\title{
CONTRASTES EXTREMOS DE TERMOHIGROMETRÍA EN LA ISLA DE EL HIERRO (ISLAS CANARIAS)
}

\author{
Montserrat SALVÀ CATARINEU11,2, Joan A. LÓPEZ-BUSTINS ${ }^{1,3}$, \\ Ferran SALVADOR FRANCH ${ }^{1,2}$, Pedro A. PADRÓN PADRÓN ${ }^{4}$ \\ y Amparo CORTÉS LUCAS ${ }^{5}$ \\ ${ }^{1}$ Facultat de Geografia i Història, Universitat de Barcelona. \\ ${ }^{2}$ Grup de Recerca del Paisatge i Paleoambients a la Muntanya Mediterrània, Universitat de \\ Barcelona. \\ ${ }^{3}$ Grup de Climatologia, Universitat de Barcelona. \\ ${ }^{4}$ Cabildo de El Hierro. \\ ${ }^{5}$ Facultat de Farmàcia i Ciències de l'Alimentació, Universitat de Barcelona. \\ salva@ub.edu, jlopezbustins@ub.edu, fsalvador@ub.edu, papadron@el-hierro.org, acortes@ub.edu
}

\section{RESUMEN}

El Hierro es la isla más occidental del archipiélago canario y se halla en latitudes subtropicales $\left(27,5^{\circ} \mathrm{N}\right)$. El clima subtopical/tropical canario de la isla se caracteriza por tener gran diversidad de microclimas debido a la importante influencia de algunos factores geográficos. El objetivo principal de este trabajo es analizar los datos de temperatura y humedad relativa del aire, registrados a resolución horaria por sensores instalados en sabinares de El Hierro, a lo largo de tres ciclos anuales (de septiembre de 2012 a agosto de 2015) y estudiar episodios extremos de termohigrométricos. Los datos provienen de dos enclaves con orientación y altitud contrastadas: El Júlan (996 $\mathrm{m}$, en la vertiente sur y en el interior) y Sabinosa (258 m, en la cara norte y cerca de la línea de costa). Los resultados obtenidos muestran ciclos termohigrométricos muy similares entre ambas áreas de estudio, pero presentan grandes diferencias en el rango de sus valores a pesar de hallarse a menos de $4 \mathrm{~km}$ de distancia horizontal. Los contrastes extremos de temperatura acontecen en julio y agosto cuando El Júlan registra hasta $21^{\circ} \mathrm{C}$ más que Sabinosa a la misma hora. Son situaciones sinópticas en que los alisios soplan fuerte y dejan Sabinosa bajo el mar de nubes. Los contrastes extremos de humedad tienen lugar también en verano, sobre todo en agosto, bajo advecciones del este de origen sahariano, donde los valores de El Júlan pueden caer por debajo del 5\% y los de Sabinosa mantenerse por encima del 90\%.

Palabras clave: Bioclimatología, El Hierro, Extremos Climáticos, Termohigrometría, Variabilidad Climática Espacial.

\begin{abstract}
El Hierro is the westernmost island of Canary Isles and it is located in the north subtropic $\left(27.5^{\circ} \mathrm{N}\right)$. The subtropical-tropical Canary climate is featured by multiple microclimates due to geographical factors. The main objective of the present study is to analyse air temperature and relative humidity data of Juniperus turbinata forests and study extreme events of thermohygrometric differences. Sensors in the branches of Juniperus turbinata trees hourly recorded these data along three annual
\end{abstract}


cycles (from September 2012 to August 2015). Data come from two locations with different aspect and altitude: El Júlan (996 masl, in the south face and inland) and Sabinosa (258 masl, in the north face and next to the sea). The results show similar thermohygrometric cycles in both locations. Despite these two points are very close (horizontally separated by $4 \mathrm{~km}$ ), a large difference is found between their respective ranges of values. The largest temperature differences take place in July and August. El Júlan can record $21^{\circ} \mathrm{C}$ more than Sabinosa at the same time in summer. Trade winds contribute to this large temperature difference because they form orographic clouds over the north face of the island (Sabinosa is to windward). The largest differences of relative humidity take place in summer too, above all, in August. Easterly winds from Sahara Desert reduce humidity in El Júlan below 5\%, whereas humidity in Sabinosa can keep over $90 \%$.

Key words: Bioclimatology, Climate Extremes, El Hierro, Spatial Climate Variability, Thermohygrometry.

\section{INTRODUCCIÓN}

En la isla de El Hierro, según Köppen, se dan cuatro tipos de climas: desértico cálido semiisotermo con alta humedad relativa, estepario cálido semiisotermo con alta humedad relativa, mediterráneo de verano cálido semiisotermo y mediterráneo de verano fresco y templado con nieblas (López Gómez y López Gómez, 1979).

El estudio de la bioclimatología de El Hierro es indispensable para determinar las variables de temperatura y humedad que condicionan la distribución geográfica del sabinar. El sabinar es un tipo de bosque termófilo que en la actualidad se localizan en áreas alejadas de las poblaciones y en dónde no existen estaciones meteorológicas. En las Islas Canarias hay dos especies de sabinas silvestres: Juniperus cedrus W.B. y Juniperus turbinata Guss. La sabina Juniperus turbinata Guss. es un endemismo mediterráneo-macaronésico que en las Islas Canarias se halla en Tenerife, Gran Canaria, La Palma, La Gomera y El Hierro (Hansen y Sundig, 1993). En concreto las formaciones de esta sabina cubren en la actualidad áreas importantes en las islas de La Gomera y de El Hierro (Kämmer, 1976), en cambio en la isla de La Palma son poco abundantes y se encuentran muy localizadas en las islas de Tenerife y Gran Canaria. Por ello es necesario conocer las condiciones climáticas en las que vive esta especie que ha visto reducida su extensión en todas las islas.

Para la conservación y gestión de esta especie es fundamental obtener información precisa sobre las condiciones climáticas donde habitan los sabinares de la Isla. Cabe destacar entre los estudios previos las tesis de Gaisberg (2005) y Stierstorfer (2005) y las investigaciones de Hernández-Padrón et al. (1985), Fernández Palacios et al. (1992), Padrón Padrón (1992), Del Arco et al., (1996), Stierstorfer y Gaisberg (2006) y Fernández Pello et al. (2006) que han trabajado con los datos existentes y alguno de ellos han aportado datos nuevos al conocimiento de la climatología y meteorología de El Hierro.

Este trabajo tiene como objetivo principal analizar los datos de temperatura y humedad relativa del aire comparando los datos de dos estaciones instaladas en el mismo tipo de bosque para comparar los contrastes termohigrométricos de cada estación y entre las dos áreas de estudio. Los sensores instalados tiene una resolución horaria y se analizan tres ciclos anuales (de septiembre de 2012 a agosto de 2015). 


\section{MÉTODOS}

\section{1. Área de estudio}

En El Hierro se encuentra actualmente el sabinar más extenso de Juniperus turbinata de todas las Islas Canarias, en concreto en la zona occidental de la isla. La sabina es uno de los elementos característicos de los bosques termófilos canarios, con un elevado valor histórico, paisajístico, ecológico y biogeográfico.

La isla de El Hierro es la más occidental del archipiélago de las Islas Canarias localizada entre $27^{\circ} 38^{\prime}-27^{\circ} 50^{\prime} \mathrm{N}$ y $17^{\circ} 53^{\prime}-18^{\circ} 09^{\prime}$, la más pequeña $\left(268,71 \mathrm{~km}^{2}\right)$ de las siete islas principales y la más joven, formada en tres etapas volcánicas entre 1,1 Ma y 2,5 ka. La altura máximas es de $1.502 \mathrm{msnm}$ en Malpaso (figura 1). El relieve y su situación ceánica permite que se den dos subtipos de clima: el litoral y el de mar de nubes (Martín y Olcina, 2001). Esto da a la isla una riqueza biológica en la que se puede observar tabaibales y cardonales, bosques termoesclerófilos como los sabinares, monteverde y pinares. Desde enero de 2000, la isla fue declarada Reserva de la Biosfera.

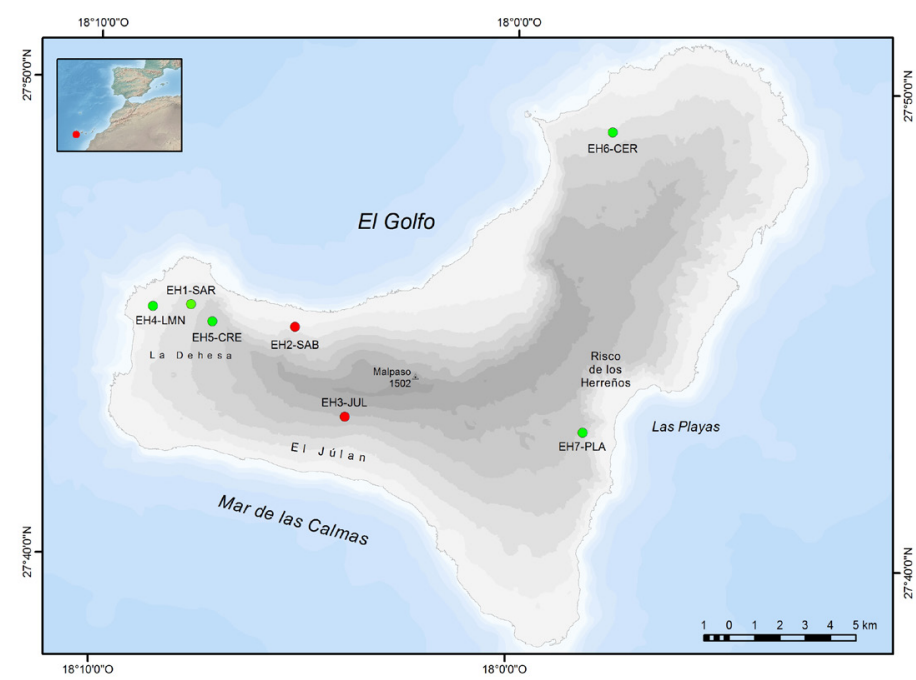

Fig. 1: Localización de las estaciones instaladas para el proyecto JUNITUR en El Hierro. En rojo las localizaciones de este estudio. Base topográfica: IGN.

\subsection{Datos climáticos}

El estudio se inició en el año 2012, año de inicio del proyecto JUNITUR, con la instalación de tres equipos de toma de datos de temperatura y humedad del aire (Ta, HR) y del suelo (Ts, Hs). En este trabajo se presentan los tres primeros años de datos de Ta y de HR de dos estaciones, una en el sabinar húmedo de Sabinosa a $258 \mathrm{~m}$ con orientación NNW y otra en el sabinar seco de El Júlan a 996 m y SSE (figura 1).

No ha sido posible obtener datos de estas áreas por otras fuentes. Hay que señalar que estos enclaves se encuentran alejados de las poblaciones de la isla y son sectores tradicionales de pastoreo (figura 2). 


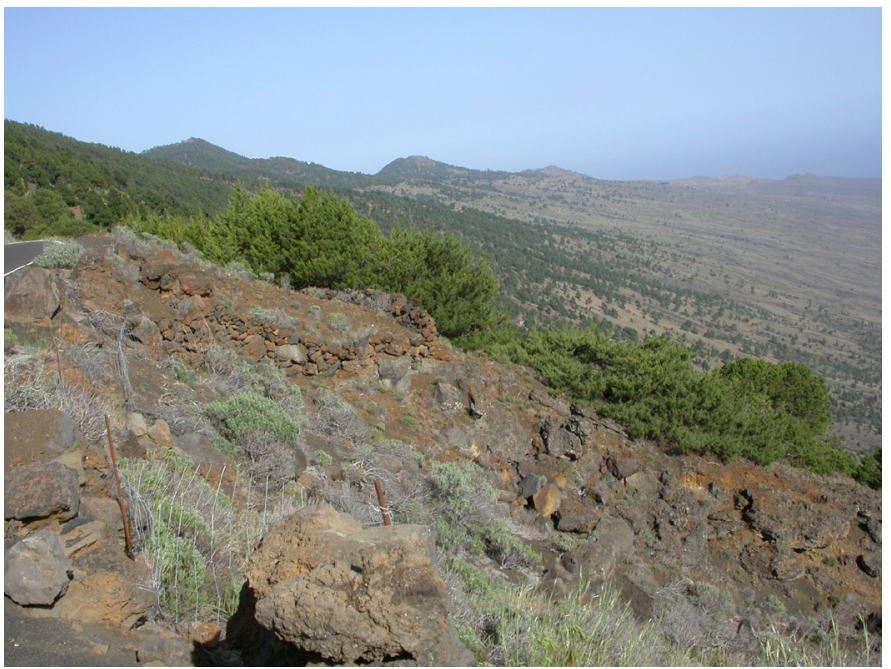

Fig. 2: Sabinar de El Júlan.

Los sensores utilizados para medir la temperatura y humedad del aire fueron U23$001 \mathrm{HOBO}{ }^{\circledR}$ Pro v2 temp/RH, que puede medir de 0 a $100 \% \mathrm{RH}, \mathrm{y}$ de $-40^{\circ} \mathrm{a}+70^{\circ} \mathrm{C} \mathrm{T}$. Todos los sensores se programaron para registrar datos a resolución horaria, con ello se obtuvo 24 registros al día/sensor (figura 3).

Todos los datos se analizaron estadísticamente para estudiar las condiciones ambientales extremas en las que viven los sabinares más occidentales de las Islas Canarias.

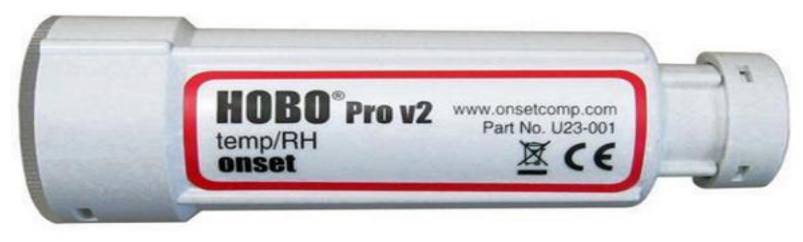

Fig. 3: Termohigrómetro del aire U23-001 HOBO® Pro v2 Temp/RH.

\section{RESULTADOS Y DISCUSIÓN}

\subsection{Temperatura}

La temperatura media del aire (Ta) para los tres años analizados (figura 4) es de $16,5^{\circ} \mathrm{C}$ en El Júlan (vertiente SSE) y de $19,2^{\circ} \mathrm{C}$ en Sabinosa (vertiente N). Los valores extremos se observaron en El Júlan con una máxima absoluta de $42,7^{\circ} \mathrm{C}$ el 8 julio de 2013 y una mínima absoluta de $5,4^{\circ} \mathrm{C}$ registrada el 15 febrero de 2014 . La oscilación máxima diaria fue de $27,6^{\circ} \mathrm{C}$ el 8 de julio de 2013 . Por otra parte, en Sabinosa la Ta máxima de $32,8^{\circ} \mathrm{C}$ se registró el 13 de agosto de 2015 y la Ta mínima de $10,6^{\circ} \mathrm{C}$ el 9 de enero de 2014. La oscilación máxima diaria fue de $10,8^{\circ} \mathrm{C}$ el 13 de agosto de 2015 . 


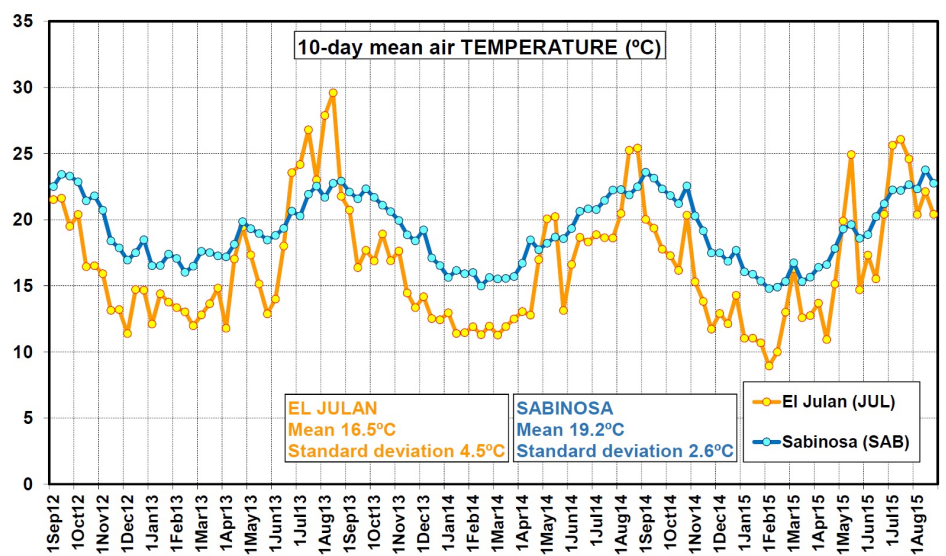

Fig. 4: Temperaturas medias del aire en periodos de 10 días en las parcelas de El Júlan y Sabinosa.

Los contrastes extremos de altas temperaturas se observaron en julio y agosto (figura 5, izquierda), cuando El Júlan registró $21,5^{\circ} \mathrm{C}$ más que Sabinosa a la misma hora el 8 de julio de 2013. Son situaciones sinópticas en que los alisios soplan fuerte y dejan Sabinosa bajo el mar de nubes (figura 5, derecha).

Los episodios extremos fríos, donde Sabinosa registra una temperatura mayor que El Júlan, son más frecuentes en mayo y junio. Se dieron en situaciones sinópticas de advección de aire frío en altura. El anticiclón de las Azores se halla desplazado a latitudes más altas y la depresión, que se encuentra en el sur peninsular, impulsa la entrada de aire frío hacia las Islas Canarias. La máxima diferencia de temperatura tuvo lugar el 22 de marzo de 2015 con un valor de $11,5^{\circ} \mathrm{C}$, donde El Julán registró $8,1^{\circ} \mathrm{C}$ y Sabinosa $19,6^{\circ} \mathrm{C}$.

\begin{tabular}{|l|c|c|}
\hline & Calor & Frío \\
\hline Enero & 0 & 1 \\
\hline Febrero & 0 & 0 \\
\hline Marzo & 0 & 3 \\
\hline Abril & 1 & 9 \\
\hline Mayo & 9 & 11 \\
\hline Junio & 6 & 14 \\
\hline Julio & 23 & 3 \\
\hline Agosto & 11 & 1 \\
\hline Septiembre & 0 & 3 \\
\hline Octubre & I0 & 2 \\
\hline Noviembre & 0 & 1 \\
\hline Diciembre & 0 & 2 \\
\hline & $\mathbf{5 0}$ & $\mathbf{5 0}$ \\
\hline
\end{tabular}

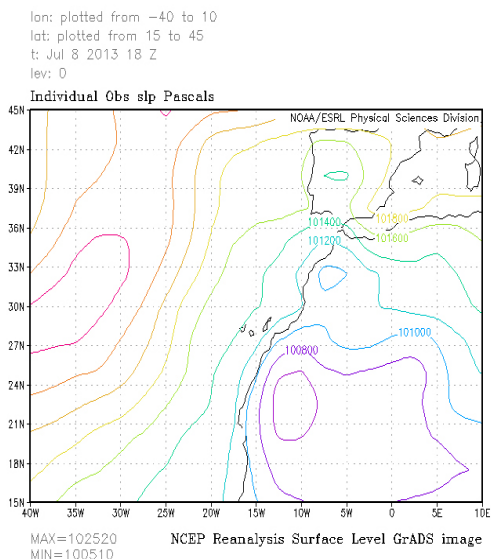

Fig. 5: Izquierda, los 50 episodios más extremos de calor y frío de El Júlan respecto a Sabinosa. Derecha, la situación sinóptica en superficie del 8 de julio de 2013. Fuente: NCEP Reanalysis Surface Level (NOAA). 


\subsection{Humedad}

La humedad relativa (HR) del aire por períodos de diez días en las dos parcelas tiene una media de $66,2 \%$ en El Júlan y 76,6\% en Sabinosa. La humedad ha registrado mayor amplitud y variabilidad en el aire de El Júlan (figura 6).

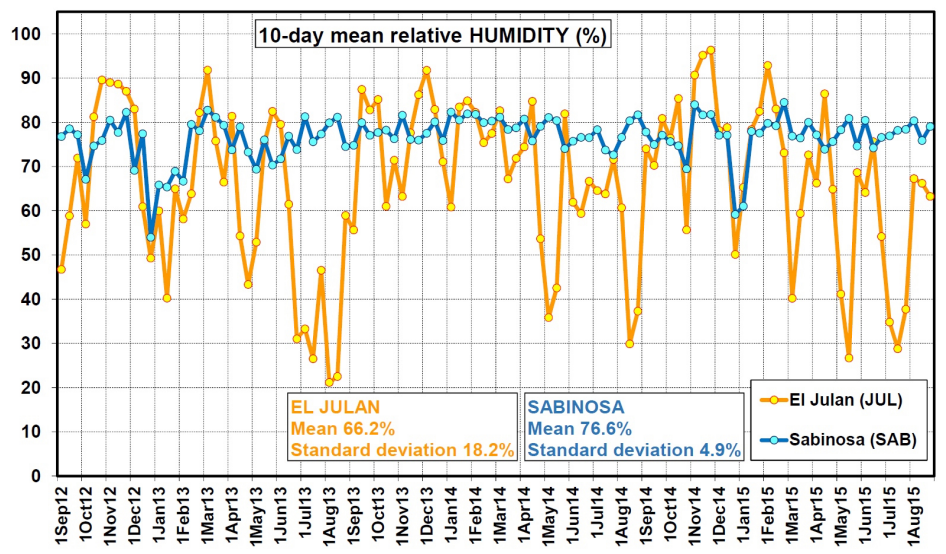

Fig. 6: Medias de la humedad del aire en periodos de 10 días en las parcelas de El Júlan y Sabinosa.

La HR del aire alcanzó 100\% en las dos parcelas. En El Júlan la HR máxima absoluta de todos los meses estuvo por encima del 90\% y la HR mínima absoluta fue de 1,2\% registrada el 4 agosto de 2013. La oscilación máxima diaria fue del 92,2\% el dia 1 de abril de 2013. En Sabinosa la HR máxima absoluta también estuvo todos los meses entre el $90 \%$ y $100 \%$ y la HR mínima absoluta fue de $10,5 \%$ el 31 de enero de 2013. La oscilación máxima diaria fue de $72,1 \%$ el 15 de julio de 2015.

Los contrastes extremos de humedad elevada entre El Júlan y Sabinosa se registraron en junio, octubre y diciembre (figura 7, izquierda), con predominio de las advecciones de componente sur. Por ejemplo, el 10 de en diciembre de 2013 se dio una diferencia de 45,2\% entre El Júlan (83,8\%) y Sabinosa (38,6\%). Los episodios extremos secos se dan a finales de primavera y en verano, destacan agosto y mayo. Se dan bajo advecciones del este de origen sahariano con la entrada de una masa de aire seco y cálido (figura 7, derecha). El 11 de mayo de 2015 en El Júlan se registró una HR de 4,1\% a las 02:00h a la misma hora en Sabinosa era de 91,4\% (figura 7).

En El Júlan los meses de verano presentan una humedad muy reducida por su orientación SSE y puede atribuirse, en parte, al efecto Foehn, ya que se observa también un incremento considerable de las temperaturas. Las condiciones de falta de humedad (mínima extrema de 1,2\%) que sufren las poblaciones de sabinares de la vertiente sur en comparación con aquéllas de la cara norte se ven reflejadas en las medias higrométricas tomadas en el campo. En verano los sabinares del vertiente sur presentan una humedad inferior y una temperatura superior alcanzando valores de $42,7^{\circ} \mathrm{C}$. 


\begin{tabular}{|l|c|c|}
\hline & Seco & Húmedo \\
\hline Enero & 1 & 3 \\
\hline Febrero & 0 & 1 \\
\hline Marzo & 3 & 2 \\
\hline Abril & 6 & 5 \\
\hline Mayo & 12 & 5 \\
\hline Junio & 4 & 10 \\
\hline Julio & 9 & 3 \\
\hline Agosto & 14 & 1 \\
\hline Septiembre & 1 & 2 \\
\hline Octubre & 0 & 7 \\
\hline Noviembre & 0 & 4 \\
\hline Diciembre & 0 & 7 \\
\hline & $\mathbf{5 0}$ & $\mathbf{5 0}$ \\
\hline
\end{tabular}

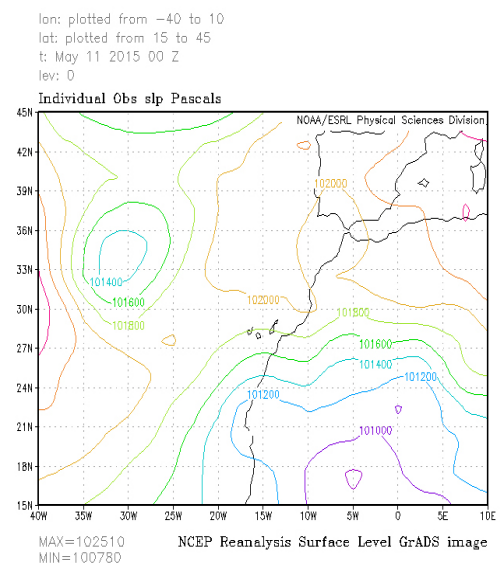

Fig. 7: Izquierda, los 50 episodios más extremos de sequedad y humedad de El Júlan respecto a Sabinosa. Derecha, la situación sinóptica en superficie del 11 de mayo de 2015. Fuente:

NCEP Reanalysis Surface Level (NOAA).

\section{CONCLUSIONES}

Los resultados de la temperatura del aire muestran que las dos estaciones se localizan en pisos bioclimáticos diferentes en función de los termotipos (suma de las temperaturas medias de los meses con temperaturas medias superiores a $0^{\circ} \mathrm{C}$ ). Según los datos obtenidos para este período de estudio (2012-2015) Sabinosa es termomediterráneo y El Júlan es mesomediterráneo.

Los contrastes extremos de temperatura acontecen en julio y agosto cuando El Júlan registra hasta $21^{\circ} \mathrm{C}$ más que Sabinosa a la misma hora. Son situaciones sinópticas en que los alisios soplan fuerte y dejan Sabinosa bajo el mar de nubes.

Los contrastes extremos de humedad entre las dos estaciones se dieron el $11 \mathrm{de}$ mayo de 2015 a las 2:00 de la noche con registros de 4,1\% (El Júlan) y de 91,4\% (Sabinosa). A parte de episodios concretos, en general los mayores contrastes tienen lugar en verano, sobre todo en agosto, bajo advecciones del este de origen sahariano, cuando los valores de El Júlan pueden caer por debajo del 5\% y los de Sabinosa mantenerse por encima del $90 \%$.

Por una parte muestran ciclos termohigrométricos muy similares y por otra presentan grandes diferencias en el rango de sus valores a pesar de hallarse a menos de 4 $\mathrm{km}$ de distancia horizontal.

Este trabajo supone un avance para el estudio de la biodiversidad de los sabinares ya que en la actualidad no había estaciones en funcionamiento en estos bosques termófilos. En el análisis de los datos de este estudio se observa que existen diferencias termohigrométricas significativas entre las dos vertientes de El Hierro donde se encuentran los sabinares.

En este trabajo preliminar se presentan los resultados de tres años, pero serán necesarios datos de otras estaciones y series más largas para calcular adecuadamente la termohigrometría de los sabinares. Además de las dos estaciones, en la actualidad 
se han incorporado otras cinco estaciones que completarán los datos de Ta y HR de este bosque termófilo Para el cálculo de algunos índices bioclimáticos es preciso tener los datos de precipitación de los sabinares, pero de momento no se han instalado hidrógrafos.

Los resultados de esta investigación permiten avanzar en el conocimiento de la bioclimatología de los sabinares de la isla más occidental de las Islas Canarias donde no existían datos de temperatura y humedad del aire. En futuros trabajos se analizarán los datos obtenidos con la clasificación bioclimática de Rivas-Martínez et al. (2011).

\section{AGRADECIMIENTOS}

Agradecemos el soporte de la Consejería de Medio Rural y Marino y Recursos Hidráulicos y a Miguel A. Rodríguez Domínguez del Cabildo insular de El Hierro por las facilidades dadas en la ejecución del trabajo de campo. Este estudio ha sido financiado por los proyectos de investigación JUNITUR (CSO2011-24425, 2012-2015) y JUNITUR+ (CSO2015-68500- R, 2016-2019) de la Secretaría de Investigación, Desarrollo e Innovación del Ministerio de Economía y Competitividad

\section{REFERENCIAS}

Del Arco Aguilar, M.J.; Acebes, J.R. y Pérez de Paz, P.L. (1996). Bioclimatology and climatophilous vegetation of the Island of El Hierro (Canary Islands). Phytoceonologia, 26, pp. 445-479.

Fernández-Palacios, J.M. et al. (1992). "Climatic responses of plant species on Tenerife, The Canary Islands". Journal of Vegetation Science, 3, pp. 595-602.

Fernández-Pello, L.; González, M.L. y Quirantes González, F. (2006). "La influencia de los factores geográficos en la organización interna de un bosque residual: el Sabinar de la Dehesa (El Hierro, Islas Canarias)". Actas III Coloquio Intern. sobre sabinares y enebrales, pp. 263-271. Soria.

Gaisberg, M. von (2005). Die Vegetation der Fußstufe von El Hierro (Kanarische Inseln). Dissertationes Botanicae, 395. J. Cramer, Berlin-Stuttgart. 364 pp.

Hansen, A. y Sunding, P. (1993). "Flora of Macaronesia. Check-list of Vascular Plants. 4 revised edition". Sommerfeltia, 17, pp. 1-295.

Hernández-Padrón, C.; Pérez de Paz, P.L. y Wildpret de la Torre, W. (1985). "Contribución al estudio bioclimatológico de El Hierro (Islas Canarias)". Vieraea, 14, pp. 77-111.

Kämmer, F. (1976): The influence of man on the vegetation of the island of Hierro. In: Kunkel, G: (ed.). Biogeography and ecology in the Canary Islands. W. Junk. The Hague.

López Gómez, J. y López Gómez, A. (1979). El clima de Canarias según la clasificación de Köpen. Estudios geográficos, 40, pp. 321-340.

Martín-Vide, J. y Olcina-Cantos, J. (2001). Climas y Tiempos en España. Madrid: Alianza Editorial.

Padrón Padrón, P.A. (1992). Estudio edafoambiental de la isla de El Hierro. Universidad de La Laguna. Facultad de Biología. Tesis Doctoral. 
Rivas-Martínez S, Rivas S, Penas A (2011) Worldwide bioclimatic classification system. Global Geobotany 1:1-634

Stiersstorfer, Ch. (2005). The Vascular Plant Vegetation in the Forest Belt of El Hierro (Canary Islands). Dissertationes Botanicae, 393. J.Cramer. Berlin-Stuttgart. 392 pp.

Stiersstorfer, Ch. y Gaisberg, M. (2006). Annotated checklist and distribution of the vascular plants of El Hierro, Canary Islands, Spain. Englera, 27, pp. 1-217. 